\title{
MRI features of growth hormone deficiency in children with short stature caused by pituitary lesions
}

\author{
CHAO XU, XINXIAN ZHANG, LINA DONG, BIN ZHU and TAO XIN \\ Department of Radiology, Xuzhou Children's Hospital, Xuzhou, Jiangsu 221002, P.R. China
}

Received July 25, 2016; Accepted March 21, 2017

DOI: $10.3892 /$ etm.2017.4377

\begin{abstract}
We verified the advantages of using magnetic resonance imaging (MRI) for improving the diagnostic quality of growth hormone deficiency (GHD) in children with short stature caused by pituitary lesions. Clinical data obtained from 577 GHD patients with short stature caused by pituitary lesions were retrospectively analyzed. There were 354 cases $(61.3 \%)$ with anterior pituitary dysplasia; 45 cases $(7.8 \%)$ of pituitary stalk interruption syndrome (PSIS); 15 cases $(2.6 \%)$ of pituitary hyperplasia due to primary hypothyroidism; 38 cases $(6.6 \%)$ of Rathke cleft cyst; 68 cases $(11.8 \%)$ of empty sella syndrome; 16 cases $(2.8 \%)$ of pituitary invasion from Langerhans cell histiocytosis; 2 cases $(0.3 \%)$ of sellar regional arachnoid cyst and 39 cases $(6.8 \%)$ of craniopharyngioma. MRI results showed that the height of anterior pituitary in patients was less than normal. Location, size and signals of posterior pituitary and pituitary stalk were normal in anterior pituitary dysplasia. In all cases pituitary hyperplasia was caused by hypothyroidism. MRI results showed that anterior pituitary was enlarged, and we detected upward apophysis and obvious homogeneous enhancement. There were no pituitary stalk interruption and abnormal signal. We also observed that after hormone replacement therapy the size of pituitary gland was reduced. Anterior pituitary atrophy was observed in Rathke cleft cyst, empty sella syndrome, sellar regional arachnoid cyst and craniopharyngioma. The microstructure of hypophysis and sellar region was studied with MRI. We detected pituitary lesions, and the characteristics of various pituitary diseases of GHD in children with short stature. It was concluded that in children with GHD caused by pituitary lesions, MRI was an excellent method for early diagnosis. This method offers clinical practicability and we believe it can be used for differential diagnosis and to monitor the therapeutic effects.
\end{abstract}

Correspondence to: Dr Xinxian Zhang, Department of Radiology, Xuzhou Children's Hospital, 18 Sudibei Road, Xuzhou, Jiangsu 221002, P.R. China

E-mail: xinxian777@yeah.net

Key words: growth hormone deficiency, magnetic resonance imaging, pituitary

\section{Introduction}

Growth hormone deficiency (GHD) refers to a condition caused by problems linked to pituitary gland in which body does not produce enough GH. Partial or complete deficiency of anterior pituitary is usually caused by receptor defects and structural abnormality. According to a study published by World Health Organization in 2000, dwarfism prevalence rate in developing countries is as high as $32.5 \%$ (1). There are only few large scale studies on the prevalence rate of dwarfism in China (2). Pituitary dwarfism caused by GHD is one of the most common conditions in children with short stature. Timely diagnosis and appropriate hormone replacement therapy can help most of the children suffering from this condition to grow-up normally. Magnetic resonance imaging (MRI) is the best diagnostic method for these children, because MRI can clearly show pituitary, pituitary stalk and adjacent structure of saddle area. There are only a limited number of comparative studies reporting on GHD in children caused by pituitary lesions (3). We conducted a retrospective analysis on clinical data obtained from 577 patients with short stature caused by pituitary lesions.

\section{Materials and methods}

Clinical data. From May 2013 to January 2016, 577 children with short stature who underwent hypophysis MRI examination and were diagnosed with GHD and had complete image data were included in this study. There were 353 males and 224 females (male, female ratio was 1.58:1). Age range was from 2 to 24 years (average, 8.1 years). All patients underwent GH stimulation test with insulin and clonidine or levodopa, while 15 cases underwent thyroid hormone detection. Diagnosis standards for GHD (4) were: i) The height was below the third percentiles or two standard deviations of the height in healthy children; ii) annual growth velocity of $<7 \mathrm{~cm} /$ year (below 3 years), $<5 \mathrm{~cm} /$ year (from 3 years to puberty) and $<6 \mathrm{~cm} /$ year (puberty); iii) childish face, short and small symmetry; iv) normal intelligence; v) bone age falls behind actual age; vi) the peak of GH in two pharmacologic provocative tests was $<10 \mu \mathrm{g} / 1$ and vii) serum insulin-like growth factor 1 was lower than the normal level. 
USA). The 8-channel craniocerebral coil was used and in all patients we applied MRI plain scan for sellar region. In 223 patients we applied Cube dynamic contrast enhancement examination. MRI plain scan sequences included sagittal view $\mathrm{T} 1$ and coronal view $\mathrm{T} 2$ fat suppression, with the field of view (FOV) of $20 \mathrm{~cm}$, slice thickness of $2 \mathrm{~mm}$, interlayer spacing of $0.3 \mathrm{~mm}, 9$ layers and matrix of $256 \times 256$. Sagittal view T1 sequences included TR $1,950 \mathrm{msec}$ and TE $25 \mathrm{msec}$, and coronal view T2 fat suppression sequences included TR 3,000 msec and TE $110 \mathrm{msec}$. Cube dynamic contrast enhancement examination included sagittal view, coronal view and axial view, with the field of view of $20 \mathrm{~cm}$, slice thickness of $1 \mathrm{~mm}, 40$ layers, matrix of 256x256, TR $490 \mathrm{msec}$ and TE $15 \mathrm{msec}$. The Gd-DTPA dose for intravenous injection was $0.05-0.1 \mathrm{mmol} / \mathrm{kg}$. Diffusion weighted imaging (DWI) scanning sequence was added for sellar region, with the b-value of $800 \mathrm{~s} / \mathrm{mm}^{2}$. For the sellar region in children suspected of having craniopharyngioma, craniocerebral computed tomography (CT) examination was conducted to examine the calcification. A Philips Brilliance 16-slice spiral CT unit (Philips Healthcare, DA Best, The Netherlands) was used to conduct examination, with the following scanning parameters: Tube voltage of $120 \mathrm{kV}$, tube current of $200 \mathrm{mAs}$, slice thickness of $3 \mathrm{~mm}$ and interlayer spacing of $3 \mathrm{~mm}$.

Observational index. Films were analyzed and interpreted by 2 experienced clinicians. The pituitary shape, height, diameter, the shape and thickness of pituitary stalk and presence of other abnormities in sellar region were verified. In the case of enhanced scanning image, attention was paid to observe the degree of lesion. The average height diameter in normal pituitary was $4.2 \pm 0.5 \mathrm{~mm}$ for children $1-10$ years; $5.3 \pm 0.4 \mathrm{~mm}$ in 10.1-15 years children and $5.2 \mathrm{~mm}$ in 15 years and older children (5). The diagnosis standard of pituitary stalk thickening was set at $>3 \mathrm{~mm}(6)$.

\section{Results}

Laboratory examination. The peak values of GH in all patients were $<10 \mu \mathrm{g} / \mathrm{l}$. One hundred and eighty-six patients who had peak values ranging from 5 to $10 \mu \mathrm{g} / \mathrm{l}$ were diagnosed as partial deficiency and 391 patients who had peak values below $5 \mu \mathrm{g} / \mathrm{l}$ were diagnosed as complete deficiency. In 15 patients with pituitary hyperplasia we used thyroid hormone test, and observed that thyroxin levels decreased while the levels of thyroid stimulating hormone increased. Pituitary hypothyroidism was detected in all these cases.

Imageological examination. GHD in children with short stature caused by pituitary lesions included: i) Three hundred fifty-four cases $(61.3 \%)$ with anterior pituitary dysplasia; ii) 45 cases $(7.8 \%)$ with pituitary stalk interruption syndrome (PSIS); iii) 15 cases $(2.6 \%$ ) with pituitary hyperplasia due to primary hypothyroidism; iv) 38 cases $(6.6 \%)$ with Rathke cleft cyst; (v) 68 cases (11.8\%) with empty sella syndrome; vi) 16 cases $(2.8 \%)$ with pituitary invasion from Langerhans cell histiocytosis ( $\mathrm{LCH})$; vii) 2 cases $(0.3 \%)$ with sellar regional arachnoid cyst and viii) 39 cases $(6.8 \%)$ with craniopharyngioma (Table I). Anterior pituitary dysplasia was characterized by flat pituitary, below normal height,
Table I. Cases of GHD in children with short stature (n).

\begin{tabular}{|c|c|c|c|}
\hline \multirow[b]{2}{*}{ Diseases } & \multicolumn{2}{|c|}{ Sex } & \multirow[b]{2}{*}{ Total } \\
\hline & Male & Female & \\
\hline $\begin{array}{l}\text { Anterior pituitary } \\
\text { dysplasia }\end{array}$ & 236 & 118 & 354 \\
\hline $\begin{array}{l}\text { Pituitary stalk } \\
\text { interruption syndrome }\end{array}$ & 31 & 14 & 45 \\
\hline Pituitary hyperplasia & 3 & 12 & 15 \\
\hline Rathke cleft cyst & 2 & 36 & 38 \\
\hline Empty sella syndrome & 49 & 19 & 68 \\
\hline LCH pituitary invasion & 9 & 7 & 16 \\
\hline $\begin{array}{l}\text { Sellar regional } \\
\text { arachnoid cyst }\end{array}$ & 2 & 0 & 2 \\
\hline Craniopharyngioma & 21 & 18 & 39 \\
\hline Total & 353 & 224 & 577 \\
\hline
\end{tabular}

GHD, growth hormone deficiency; LCH, Langerhans cell histiocytosis.

normal position and signal of posterior pituitary as well as no interruption, diminution or abnormal signal in pituitary stalk (Fig. 1A).

In addition to anterior pituitary dysplasia, PSIS was characterized by the absence or extremely thin pituitary stalk, high signal ectopia of posterior pituitary $\mathrm{T} 1$ and nodular shadow of high signal was found in third ventricle funnel fossae or median eminence (Fig. 1B and C). Pituitary hyperplasia was characterized by anterior pituitary enlargement, symmetric projection of superior border, no interruption or abnormal signal in pituitary stalk, obviously homogeneous enhancement after enhancement scanning and reduced volume of thyroid after replacement therapy (Fig. 1D-F). Rathke cleft cyst was characterized by sellar region, partially extending to suprasellar region, uniform signal in lesion, low signal on $\mathrm{T} 1$, high signal on T2, no enhancement of capsule wall and content in case of enhancement scanning and atrophy of anterior pituitary under compression (Fig. 2A and B).

Empty sella syndrome was characterized by empty sella turcica, taking on cerebrospinal fluid signal and atrophy of pituitary under compression (Fig. 2C and D). LCH pituitary invasion was characterized by pituitary stalk thickening or space-occupying lesion, equisignal $\mathrm{T} 1$ and $\mathrm{T} 2$, and obvious enhancement a, normal size and signal of anterior pituitary, and high signal of posterior pituitary lost on T1 (Fig. 2E and F). Sellar regional arachnoid cyst usually occurs in parasellar region, however, in a few cases it was detected in intrasellar region. It was characterized by round or oval shape, regular morphology, basically same signal with cerebrospinal fluid signal, low signal of DWI sequence, atrophy of pituitary under compression, and no enhancement of the capsule wall and content in case of enhancement scanning (Fig. 3A-C).

Craniopharyngioma had complex MRI signal, heterogeneous enhancement of tumor in case of enhancement scanning, enhancement of complete or partial capsule wall, atrophy of pituitary under compression, and lesions mostly accompanied 

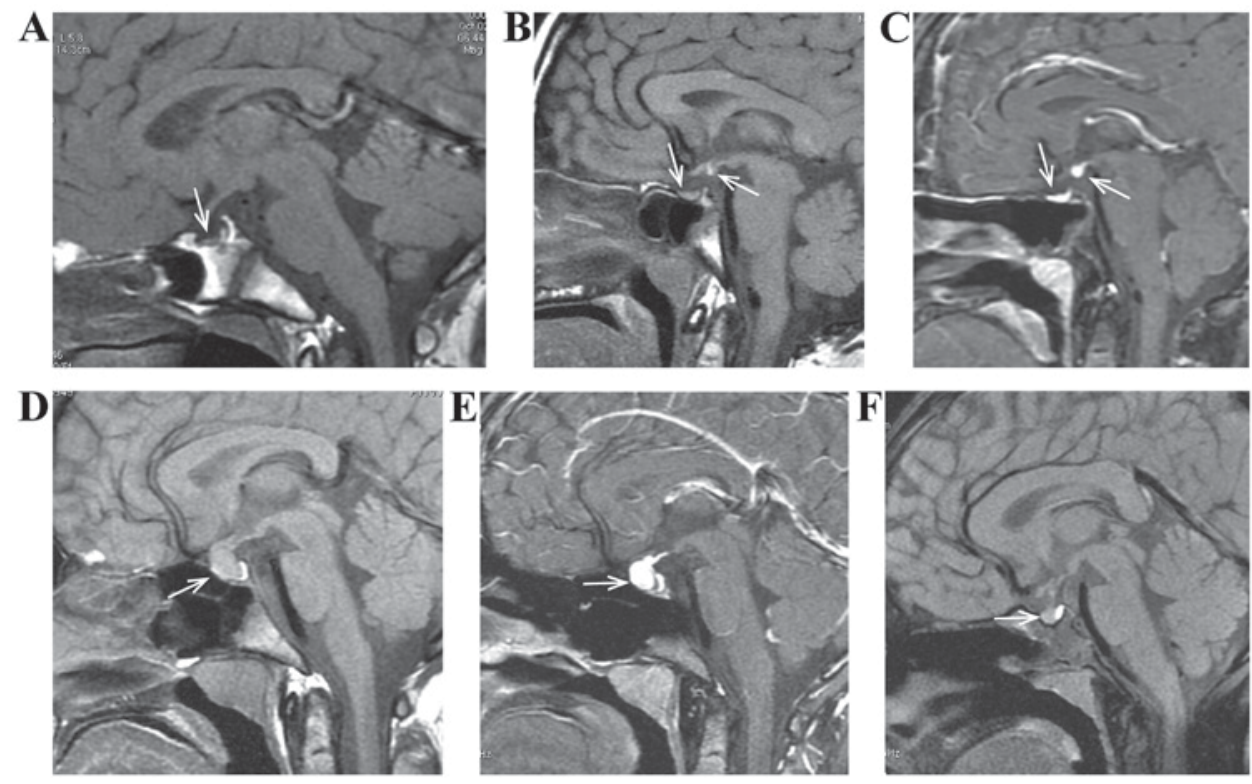

Figure 1. (A) Male, 5 years of age, anterior pituitary dysplasia. He had 'growth retardation and short stature', characterized by thin and small anterior pituitary and the existence of high signal of posterior pituitary. (B and C) Female, 6 years of age, pituitary stalk interruption syndrome. She had 'growth retardation and short stature', characterized by thin and small anterior pituitary, no pituitary stalk and the existence of high signal of posterior pituitary T1. (D-F) Female, 10 years of age, pituitary hyperplasia due to primary hypothyroidism. She had 'growth retardation', characterized by anterior pituitary enlargement and uniform enhancement in case of enhancement scanning. (F) Pituitary of a patient returned to normal size after treatment by re-examination of the magnetic resonance imaging.
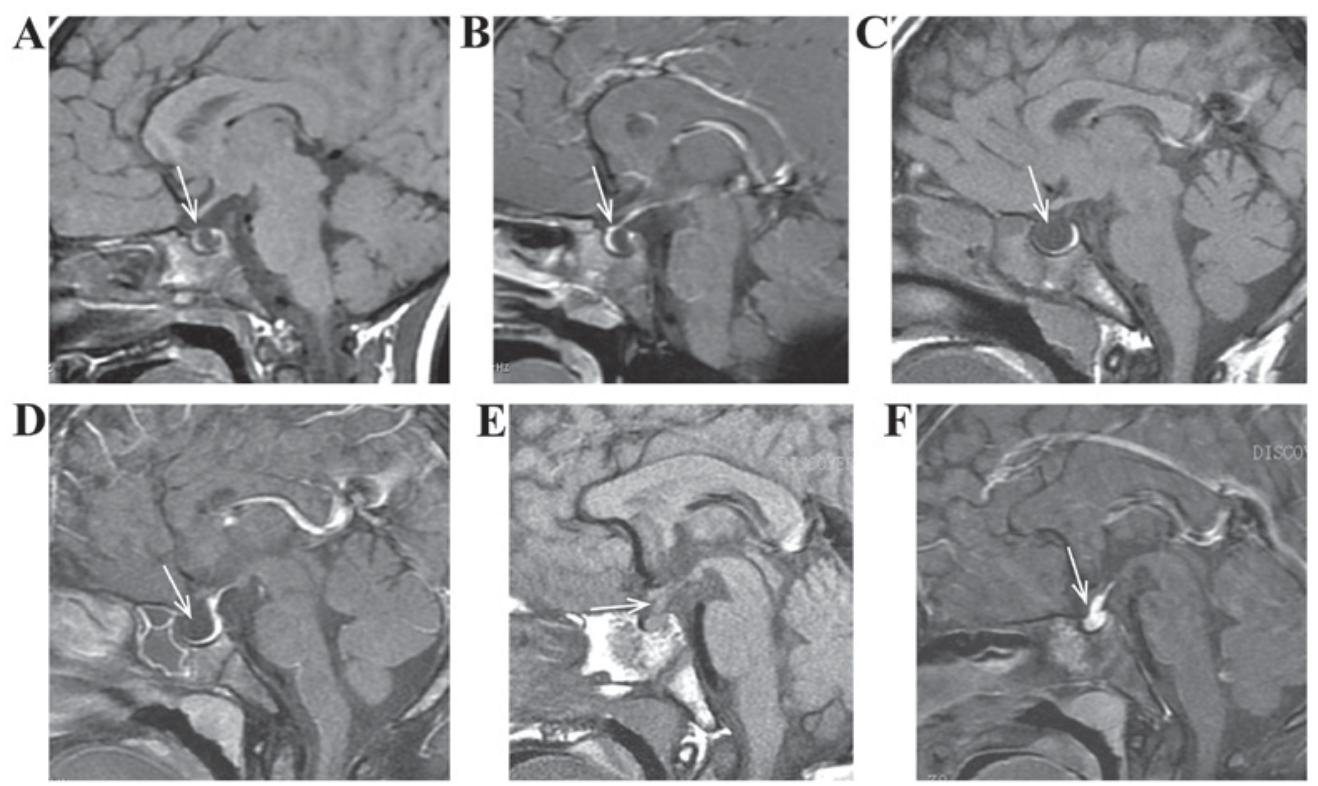

Figure 2. (A and B) Female, 7 years of age, Rathke cleft cyst. She had 'growth retardation and short stature', characterized by cystic space-occupying between anterior pituitary and posterior pituitary, low signal of T1, atrophy of anterior pituitary under compression, and no enhancement of capsule wall and content in case of enhancement scanning. (C and D) Male, 9 years of age, empty sella syndrome. He had 'growth retardation and short stature', characterized by empty sella turcica, taking on cerebrospinal fluid signal and atrophy of pituitary under compression. (E and F) Male, 6 years of age, LCH pituitary invasion. He had 'polydipsia polyuria and short stature', characterized by normal size and signal of anterior pituitary, high signal of posterior pituitary lost on T1, pituitary stalk thickening and obvious enhancement. LCH, Langerhans cell histiocytosis.

by calcification in case of craniocerebral CT examination, especially eggshell calcification (Fig. 3D-F).

\section{Discussion}

Short stature means that under the same conditions, the height of child is below normal (same race, same region, same sex and same age child by more than two standard deviations or below the third percentile of growth curve of normal child) (7). With social progress and improvement of medical level, people pay more attention to their heights. Pituitary dwarfism caused by GHD is the most common pathogenesis in children with short stature that can negatively affect children's health. Timely diagnosis and treatment with appropriate hormone replace- 

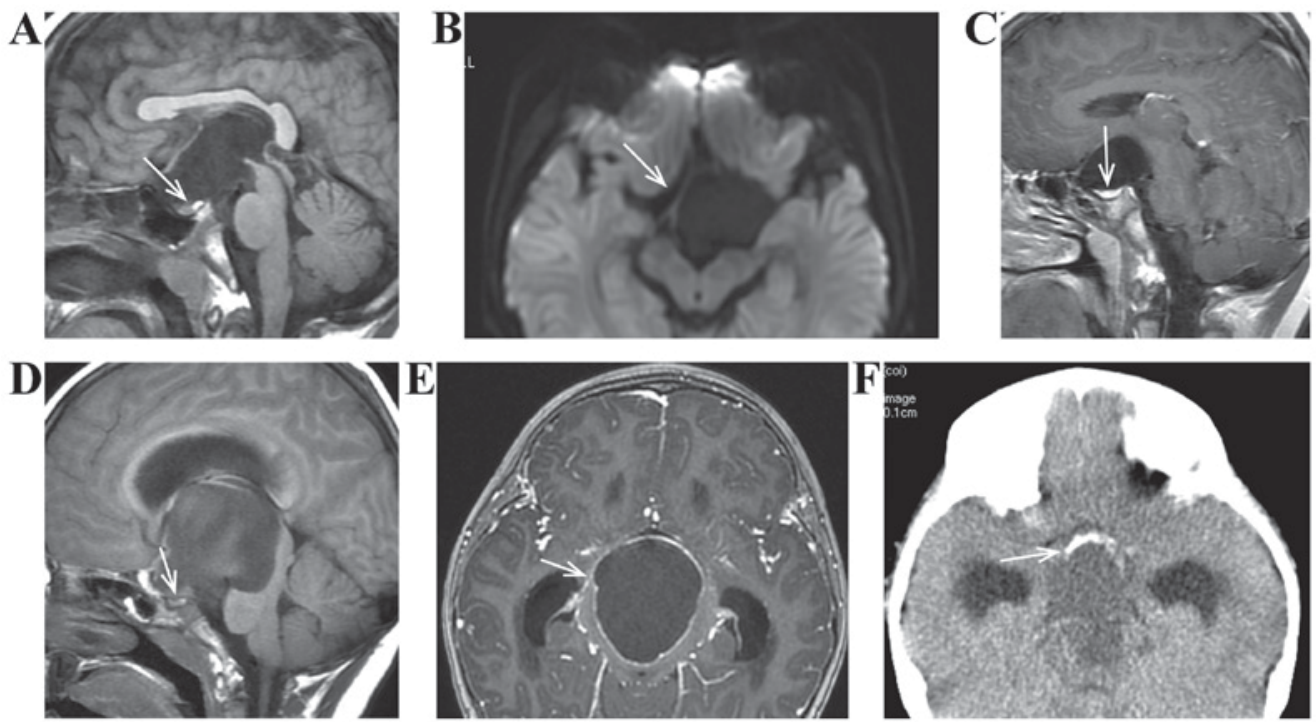

Figure 3. (A-C) Male, 10 years of age, sellar regional arachnoid cyst. He had 'growth retardation and short stature', characterized by atrophy of sellar region close to pituitary under compression, basically same signal with cerebrospinal fluid signal, low signal of DWI sequence, and no enhancement of capsule wall and content in case of enhancement scanning. (D-F) Female, 8 years of age, craniopharyngioma. She had 'growth retardation and blurred vision', characterized by atrophy of sellar region close to pituitary under compression and obvious enhancement of capsule wall in case of enhancement scanning. (F) Eggshell calcification of lesion. DWI, diffusion weighted imaging.

ment therapy can help most children to grow up normally (8). Early detection, early diagnosis and early treatment have an important significance for the prognosis and improvement of life quality for patients with short stature.

Located at ventral hypothalamus, pituitary is the most complicated endocrine gland. Hormones generated by pituitary are related to the growth of bones and soft tissues, it also affects the activities of the endocrine glands. The size and the shape of pituitary gland are affected by sex, age and internal environment. Any change in its size and shape can directly influence the secretion level of pituitary hormone (9). Prior studies showed that children with abnormal MRI pituitary images are obviously shorter and respond better to hormone replacement therapy $(10,11)$. The cause of anterior pituitary dysplasia is not clear, however, the reduction of blood supply for pituitary gland or anoxia asphyxia in newborns are probably involved in the development of this condition (12).

We had 354 (61.3\%) patients (236 males and 118 females) with small anterior pituitary, subnormal height, existence of high signal of posterior pituitary and no displacement, interruption or abnormal pituitary stalk signal. PSIS is a rare congenital abnormality of the pituitary that is responsible for anterior pituitary deficiency. It is characterized by a classic triad of interrupted pituitary stalk, absent or ectopic posterior pituitary and anterior pituitary hypoplasia or aplasia. Although PSIS pathogenesis is still unclear it has been suggested that it may be related to abnormities in perinatal period or craniocerebral trauma $(12,13)$. Forty-five cases $(7.8 \%)$ including 31 males and 14 females had PSIS. Typical MRI features of PSIS included: Extremely small pituitary stalk, disappearance of high signal of posterior pituitary in pituitary fossa and anterior pituitary dysplasia.

Since thyroxin is the stimulating factor for the synthesis of $\mathrm{GH}$, thyroid hypofunction can negatively affect the secretion of GH (9,12-14). There were 15 cases $(2.6 \%)$ including 3 males and 12 females with the following MRI features: Anterior pituitary enlargement, symmetric projection of superior border, no interruption or abnormal signal in pituitary stalk, obviously homogeneous enhancement after enhancement scanning and reduced thyroid after replacement therapy. In 38 cases $(6.6 \%)$ including 2 males and 36 females, MRI imaging showed sellar occupying and in some cases we detected extension to suprasellar region. Usually this condition is observed in females (15). MRI results revealed low, equal or high signal on T1 and high signal on T2. No enhancement was found in capsule wall and content in case of enhancement scanning conducted by applying Gd-DTPA. Empty sella syndrome was caused by subarachnoid space charging into sellar region under the impact of cerebrospinal fluid pressure (16). Sixty-eight (11.8\%) cases (49 males and 19 females), showed empty sella turcica and cerebrospinal fluid signal in most part. Two $(0.3 \%)$ male patients were detected with sellar regional arachnoid cyst which often occurs in parasellar region and sometimes in sellar region (17). Signals were basically the same as cerebrospinal fluid signal. Signals were uneven long T1 and T2 signals. Low signals were found on DWI sequence and no enhancement of capsule wall and content was found in case of enhancement scanning.

Craniopharyngioma accounted for $1.2-4.6 \%$ of intracranial tumors in children and is most common in children under 15 years. It is often cystic and cystic solid and usually appears in suprasellar region and in some cases in sellar region (18). Thirty-nine $(6.8 \%)$ patients (21 males and 18 females), had complex craniopharyngioma signal and complete or partial enhancement of the capsule wall. Craniopharyngioma was mostly accompanied by calcification and especially eggshell calcification. Dermoid cyst in sellar region is derived from ectoderm tissues, and content is mainly composed of keratin and solid crystal cholesterol, usually formed along lower plenum of arachnoid and grew slowly. Its T1 was low and T2 was generally equal or greater than cerebrospinal fluid signal. Epidermoid cyst on DWI sequence was high with no obvious enhancement of the capsule wall (19). 
Dermoid cyst in sellar region is a rare congenital tumor. Since a capsule content contains epithelial keratinization casts, sebum, sweat and hair, MRI examination often shows uneven signals on the capsule content. Usually there is fat signal and enhancement of capsule wall without enhancement of content (19). LCH pituitary invasion is a rare condition mostly seen in children which is characterized by clone-like proliferation of reticular cells and dendritic cells. This condition is universally believed to be related to immune dysfunction. MRI features associated with LCH invading hypothalamus-pituitary are pituitary stalk thickening or space-occupying lesions (20). There were 16 (2.8\%) cases ( 9 males and 7 females) with equal $\mathrm{T} 1$ and T2 signals and obvious enhancement. Size and signal of anterior pituitary were normal, and posterior pituitary was losing high signal on T1WI. It was probably due to the invasion of abnormally proliferative Langerhans cells to pituitary portal system, which caused a reduction in $\mathrm{GH}$ production and secretion.

Sellar region has a complex structure and locates in the middle of skull base, with narrow spatial position and irregular surrounding structure. MRI diagnosis value for cases of sellar region lesions has been recognized and MRI has been proved to be the best imaging method to observe pituitary structure and lesions (21). With higher signal-to-noise ratio and tissue contrast, 3.0T MRI can optimize the display of microscopic anatomical structures, improve spatial image resolution thus it is suitable to be used in study of pituitary lesions in children. With respect to the parameters of scanning sequence of MRI on sellar region, we used MRI with high field intensity in this study. We used layer thickness of $2 \mathrm{~mm}$ and interlayer spacing of $0.3 \mathrm{~mm}$ and added fat suppression sequences. Moreover, all isotropic data of the whole brain were collected through Cube dynamic enhancement examination (layer thickness of $1 \mathrm{~mm}$ ). After the completion of scanning, image reconstruction at any direction was conducted. The ultrathin layer with high signalto-noise ratio clearly displayed the microstructure of pituitary, pituitary stalk and sellar region. Partial volume effects were minimized in order to provide objective basis for the diagnosis of clinical GHD in children with short stature caused by pituitary lesions.

We concluded that pituitary dwarfism caused by GHD is one of the most common pathogenesis detected in children with short stature. Timely diagnosis can lead to early treatment which in turn can offer patients a normal growth. By virtue of higher soft tissue resolution, MRI can clearly show the pituitary microstructure and easily detect the possible lesions. It can also accurately reflect the characteristics of various pituitary diseases in GHD, and provides high clinical practicability for the studies on children with short stature caused by pituitary lesions.

\section{References}

1. de Onis M, Frongillo EA and Blössner M: Is malnutrition declining? An analysis of changes in levels of child malnutrition since 1980. Bull World Health Organ 78: 1222-1233, 2000.

2. Li R, Li H and Lang B: Epidural anaesthesia for caesarean section in pituitary dwarfism. Anaesth Crit Care Pain Med 36 123-124, 2016.
3. Wolfsdorf J, Farquhar JW and Rigal WM: Screening tests for growth-hormone deficiency in dwarfism. Lancet 2: 1271-1273, 1967.

4. Shalet SM, Toogood A, Rahim A and Brennan BM: The diagnosis of growth hormone deficiency in children and adults. Endocr Rev 19: 203-223, 1998.

5. Turner HE, Nagy Z, Gatter KC, Esiri MM, Harris AL and Wass JA: Angiogenesis in pituitary adenomas and the normal pituitary gland. J Clin Endocrinol Metab 85: 1159-1162, 2000.

6. Leger J, Velasquez A, Garel C, Hassan M and Czernichow P: Thickened pituitary stalk on magnetic resonance imaging in children with central diabetes insipidus. J Clin Endocrinol Metab 84: 1954-1960, 1999.

7. Majcher A, Bielecka-Jasiocha J and Pyrzak B: Analysis of reasons of short stature in own material. Pediatr Endocrinol Diabetes Metab 15: 152-156, 2009 (In Polish).

8. Dorantes-Alvarez L and Parra-Paredes MS: Clinical experience with the treatment of dwarfism caused by growth hormone deficiency using biosynthetic somatotropin (Somatonorm)]. Bol Med Hosp Infant Mex 46: 531-536, 1989 (In Spanish).

9. London DR: Pituitary diseases-2. Diseases of the anterior pituitary gland. Nurs Times 72: 966-968, 1976.

10. Coutant R, Rouleau S, Despert F, Magontier N, Loisel D and Limal JM: Growth and adult height in GH-treated children with nonacquired GH deficiency and idiopathic short stature: the influence of pituitary magnetic resonance imaging findings. $\mathrm{J}$ Clin Endocrinol Metab 86: 4649-4654, 2001.

11. Tsai SL, Laffan E and Lawrence S: A retrospective review of pituitary MRI findings in children on growth hormone therapy. Pediatr Radiol 42: 799-804, 2012.

12. Dekeyzer S, Herregods N, Meersschaut V and De Schepper J: Pituitary stalk interruption syndrome. JBR-BTR 96: 393, 2013.

13. Gascoin-Lachambre G, Brauner R, Duche L and Chalumeau M: Pituitary stalk interruption syndrome: diagnostic delay and sensitivity of the auxological criteria of the growth hormone research society. PLoS One 6: e16367, 2011.

14. Wolansky LJ, Leavitt GD, Elias BJ, Lee HJ, Dasmahapatra A and Byrne W: MRI of pituitary hyperplasia in hypothyroidism. Neuroradiology 38: 50-52, 1996.

15. Billeci D, Marton E, Tripodi M, Orvieto E and Longatti P: Symptomatic Rathke's cleft cysts: a radiological, surgical and pathological review. Pituitary 7: 131-137, 2004.

16. Ratnakaran B, Punnoose VP, Das S and Kartha A: Psychosis in secondary empty sella syndrome following a russell's viper bite. Indian J Psychol Med 38: 254-256, 2016.

17. Ildan F, Cetinalp E, Bağdatoğlu H, Boyar B and Uzuneyüoglu Z: Arachnoid cyst with traumatic intracystic hemorrhage unassociated with subdural hematoma. Neurosurg Rev 17: 229-232, 1994

18. Garnett MR, Puget S, Grill J and Sainte-Rose C: Craniopharyngioma. Orphanet J Rare Dis 2: 18, 2007.

19. Tanaka T, Yasumoto R and Kawano M: Epidermoid cyst arising from the spermatic cord area. Int J Urol 7: 277-279, 2000.

20. Makras P, Samara C, Antoniou M, Zetos A, Papadogias D, Nikolakopoulou Z, Andreakos E, Toloumis G, Kontogeorgos G, Piaditis G, et al: Evolving radiological features of hypothalamo-pituitary lesions in adult patients with Langerhans cell histiocytosis (LCH). Neuroradiology 48: 37-44, 2006.

21. Sade B, Mohr G and Vézina JL: Distortion of normal pituitary structures in sellar pathologies on MRI. Can J Neurol Sci 31: 467-473, 2004. 